\title{
ON THE RECURRENCE OF A CERTAIN CHAIN ${ }^{1}$
}

D. A. DARLING AND P. ERDÖS

Let balls be placed successively and independently in urns $U_{1}$, $U_{2}, \cdots$, urn $U_{i}$ receiving each ball with probability $p_{i}, i=1,2, \cdots$. After $n$ balls have been placed let $L_{n}$ be the number of urns containing an odd number of balls. The event $\left[L_{n}=0\right.$ for infinitely many $\left.n\right]$ has probability one or zero, termed respectively the "recurrent" and the "transient" cases. In $[1$, p. 94] it was stated that "it seems impossible to obtain a general criterion in terms of $\left\{p_{k}\right\}$ to ensure the recurrent case," and in [2] it was stated "it would appear that the necessary and sufficient conditions are rather delicate and not to be exhibited in neat form."

In this note we clarify matters, showing that the condition (1) given below, previously known to be sufficient for recurrence ([1] and [2]), is also necessary.

Without loss of generality we assume $p_{i}>0, i=1,2, \cdots, p_{1} \geqq p_{2}$ $\geqq p_{3} \geqq \cdots$, and we set $f_{n}=p_{n}+p_{n+1}+\cdots$, so that $f_{1}=1$ and $f_{n}$ decreases monotonically to zero.

Theorem. A necessary and sufficient condition for recurrence is that

$$
\sum_{1}^{\infty} \frac{1}{2^{n} f_{n}}=\infty
$$

In the following $c_{1}, c_{2}, \cdots$ are suitable absolute positive constants. Let $X_{1}, X_{2}, \cdots$ be mutually independent Bernoulli random variables taking the values 0 or 1 with probabilities $\frac{1}{2}$ each, and set $S=\sum_{i=1}^{\infty} p_{i} X_{i}$. It was shown in [2] that a necessary and sufficient condition for recurrence is that $E(1 / S)=\infty$. Let $N=\min \left\{n \mid X_{n}=1\right\}$ and let $E_{n}$ be the event $[N=n]$, so that $P\left(E_{n}\right)=2^{-n}$. Since $S \leqq f_{N}$ we have $E(1 / S) \geqq E\left(1 / f_{N}\right)=\sum_{1}^{\infty} 1 /\left(2^{n} f_{n}\right)$, so that condition (1) is sufficient, and the necessity will follow if we show that $E\left(S^{-1} \mid E_{n}\right) \leqq c_{1} / f_{n}$.

Let $A_{n j}=\left[j S<f_{n}\right], j=0,1, \cdots ; n=1,2, \cdots$. We assert that it is sufficient to prove

$$
\sum_{j=0}^{\infty} P\left(A_{n j} \mid E_{n}\right) \leqq c_{2}
$$

for if (2) is true

Received by the editors November 15, 1966.

1 Research supported by a National Science Foundation Grant No. GP-3466. 


$$
\begin{aligned}
c_{2} & \geqq \sum_{j=0}^{\infty} P\left(A_{n j} \mid E_{n}\right)=\sum_{j=0}^{\infty} P\left(f_{n} / S>j \mid E_{n}\right) \\
& \geqq \int_{0}^{\infty} P\left(f_{n} / S>x \mid E_{n}\right) d x=E\left(f_{n} / S \mid E_{n}\right) \\
& =f_{n} E\left(1 / S \mid E_{n}\right) .
\end{aligned}
$$

Let now $T_{n k}=\sum_{i=n+1}^{n+k} X_{i}, n=0,1, \cdots ; k=1,2, \cdots$, so that if $E_{n}$ occurs we obtain by partial summation,

$$
\begin{aligned}
S & =\sum_{i=n}^{\infty} p_{i} X_{i} \\
& =p_{n}+\sum_{k=1}^{\infty} T_{n k}\left(p_{n+k}-p_{n+k+1}\right),
\end{aligned}
$$

and let $B_{n j}=\bigcup_{k \geq j}\left[T_{n k}<k / 4\right]$. We next assert that $A_{n j} E_{n} \subset B_{n j} E_{n}$ for $j \geqq 4 ; n=1,2, \cdots$. For suppose $\bar{B}_{n j} E_{n}$ occurs. Then

$$
\begin{aligned}
S & =p_{n}+\sum_{k=1}^{\infty} T_{n k}\left(p_{n+k}-p_{n+k+1}\right) \\
& \geqq p_{n}+(1 / 4) \sum_{k=j}^{\infty} k\left(p_{n+k}-p_{n+k+1}\right)
\end{aligned}
$$

and

$$
\begin{aligned}
f_{n} & =p_{n}+\sum_{k=1}^{\infty} k\left(p_{n+k}-p_{n+k+1}\right) \\
& =p_{n}+\sum_{k=1}^{j-1}+\sum_{k=j}^{\infty} \leqq j p_{n}+\sum_{k=j}^{\infty} k\left(p_{n+k}-p_{n+k+1}\right) .
\end{aligned}
$$

Consequently

$$
j S \geqq j p_{n}+(j / 4) \sum_{k=j}^{\infty} k\left(p_{n+k}-p_{n+k+1}\right) \geqq f_{n}
$$

for $j \geqq 4$, so that $S \geqq f_{n} / j$ and $\bar{A}_{n j} E_{n}$ occurs.

Hence $P\left(A_{n j} \mid E_{n}\right) \leqq P\left(B_{n j} \mid E_{n}\right), j \geqq 4$, and to prove (2) it is sufficient to prove $\sum q_{j}<c_{3}$, where $q_{j}=P\left(B_{n j} \mid E_{n}\right), q_{j}$ being independent of $n$.

Setting $V_{j}=T_{0 j}=X_{1}+X_{2}+\cdots+X_{j}$ we have

$$
\begin{aligned}
q_{j} & =P\left(V_{k}<k / 4 \quad \text { for some } k \geqq j\right) \\
& \leqq \sum_{k=j}^{\infty} P\left(V_{k}<k / 4\right) .
\end{aligned}
$$


The terms in the last sum are well known to decrease exponentially (cf., e.g., Chernoff [3, Theorem 1]), so that $\sum q_{j}$ converges, (2) holds, and the theorem is proved.

\section{REFERENCES}

1. F. Spitzer, Principles of random walk, Van Nostrand, New York, 1964.

2. D. A. Darling, Some limit theorems associated with multinomial trials, Proc. Fifth Berkeley Sympos. Math. Stat. and Prob., Vol. II, Part I, 1965, pp. 345-350.

3. $\mathrm{H}$. Chernoff, $A$ measure of asymptotic efficiency of tests of hypotheses based on the sums of observations, Ann. Math. Statist. 23 (1952), 493-507.

UNIVERSITY OF MichigaN 